\title{
Deficiency of $A t f 3$, an adaptive-response gene, protects islets and ameliorates inflammation in a syngeneic mouse transplantation model
}

\author{
E. J. Zmuda • M. Viapiano $•$ S. T. Grey $•$ G. Hadley $~$ \\ A. Garcia-Ocaña $\cdot$ T. Hai
}

Received: 20 August 2009/Accepted: 4 January 2010/Published online: 28 March 2010

(C) Springer-Verlag 2010

\begin{abstract}
Aims/hypothesis Islet transplantation is a potential therapeutic option for type 1 diabetes. However, the need for multiple donors per patient and heavy immunosuppression of the recipients limit its use. The goal of this study was to test whether the gene encoding activating transcription factor 3 (ATF3), a stress-inducible pro-apoptotic gene, plays a role in graft rejection in islet transplantation.

Methods We compared wild-type (WT) and Atf3 knockout (KO) islets in vitro using stress paradigms relevant to islet transplantation: isolation, inflammation and hypoxia. We also compared the WT and KO islets in vivo using a syngeneic mouse transplantation model.

Results ATF3 was induced in all three stress paradigms and
\end{abstract}

Electronic supplementary material The online version of this article (doi:10.1007/s00125-010-1696-x) contains supplementary material, which is available to authorised users.

E. J. Zmuda $\cdot$ M. Viapiano $\cdot$ T. Hai

Molecular, Cellular and Developmental Biology Program,

Ohio State University,

Columbus, OH, USA

E. J. Zmuda · T. Hai

Department of Molecular and Cellular Biochemistry,

Ohio State University,

Columbus, OH, USA

E. J. Zmuda $\cdot$ M. Viapiano $\cdot$ T. Hai

Center for Molecular Neurobiology, Ohio State University,

Columbus, $\mathrm{OH}$, USA

M. Viapiano

Department of Neurological Surgery, Ohio State University,

Columbus, OH, USA

G. Hadley

Department of Surgery, Ohio State University,

Columbus, $\mathrm{OH}$, USA played a deleterious role in islet survival, as evidenced by the lower viability of WT islets compared with $\mathrm{KO}$ islets. ATF3 upregulated various downstream target genes in a stress-dependent manner. These target genes can be classified into two functional groups: (1) apoptosis (Noxa [also known as Pmaipl] and Bnip3), and (2) immunomodulation (Tnf $\alpha$ [also known as $\operatorname{Tn} f$ ], Il-1 $\beta$ [also known as $I l l b$ ], Il-6 [also known as Il6] and Ccl2 [also known as Mcp-1]). In vivo, Atf3 KO islets performed better than WT islets after transplantation, as evidenced by better glucose homeostasis in the recipients and the reduction of the following variables in the KO grafts: caspase 3 activation, macrophage infiltration and expression of the above apoptotic and immunomodulatory genes.

\section{S. T. Grey}

Gene Therapy and Autoimmunity Group,

Garvan Institute of Medical Research,

Darlinghurst, NSW, Australia

A. Garcia-Ocaña

Department of Medicine,

Division of Endocrinology and Metabolism,

University of Pittsburgh,

Pittsburgh, PA, USA

A. Garcia-Ocaña

Department of Cell Biology and Physiology,

University of Pittsburgh,

Pittsburgh, PA, USA

T. Hai $(\square)$

Room 174 Rightmire Hall, Ohio State University,

1060 Carmack Road,

Columbus, OH 43210, USA

e-mail: hai.2@osu.edu 
Conclusions/interpretation ATF3 plays a role in islet graft rejection by contributing to islet cell death and inflammatory responses at the graft sites. Silencing the ATF3 gene may provide therapeutic benefits in islet transplantation.

Keywords ATF3 - Cell death · Inflammation · Islet transplantation · Primary non-function - Stress response · Transcription factor

$\begin{array}{ll}\text { Abbreviations } \\ \text { ATF3 } & \text { Activating transcription factor } 3 \\ \text { CCL2 } & \text { Chemokine (C-C motif) ligand } 2 \\ \text { ChIP } & \text { Chromatin immunoprecipitation } \\ \text { ßgal } & \beta \text {-Galactosidase } \\ \text { HIF1- } \alpha & \text { Hypoxia-inducible factor } 1 \text { alpha subunit } \\ \text { KO } & \text { Knockout } \\ \text { PI } & \text { Propidium iodide } \\ \text { POD } & \text { Postoperative day } \\ \text { ROI } & \text { Region of interest } \\ \text { STZ } & \text { Streptozotocin } \\ \text { TSS } & \text { Transcriptional start site } \\ \text { WT } & \text { Wild-type }\end{array}$

\section{Introduction}

Since the pioneering work demonstrating that transplantation of islets of Langerhans into diabetic rodents could normalise their blood glucose levels, islet transplantation has been proposed to be a potential treatment for type 1 diabetes [1-7]. Recent advances in human islet transplantation $[1,8]$ further strengthened this view. However, major limitations prevent islet transplantation from becoming a widespread clinical reality: (1) the requirement for large numbers of islets per patient severely reduces the number of potential recipients, and (2) the need for heavy immunosuppression significantly affects the paediatric population of patients because of their vulnerability to long-term immunosuppression. Strategies that can overcome these limitations will greatly enhance the therapeutic potential of islet transplantation.

A major reason for islet loss during transplantation is the apoptotic death of islet cells, an event caused by multiple factors that can be classified into two main groups. The first consists of those leading to 'primary non-function', a term referring to islet dysfunction and loss for reasons other than specific immune rejection by the recipients. Factors causing primary non-function include deleterious conditions that islets face during isolation and the hostile environment in the recipients such as the hyperglycaemic milieu of the hosts and the non-specific inflammatory attack by the host innate immunity, which involves macrophages and dendritic cells [9-17]. The second group of factors causing islet death in transplantation is the specific immune attacks by the host adaptive immunity, which involves $\mathrm{T}$ and $\mathrm{B}$ lymphocytes.

Although both primary non-function and specific immune attack contribute to graft failure, primary non-function is thought to be the main reason for islet failure in the early transplantation stage and for the requirement of a large number of islets (two or more pancreases per patient) to achieve euglycaemia (see Wu et al. [18] for references). Despite its importance, primary non-function is not well understood at the molecular level. In this study, we investigated the potential roles of the gene encoding activating transcription factor 3 (ATF3), a stress-inducible pro-apoptotic gene, in primary non-function. Atf3 encodes a member of the ATF/CREB family of transcription factors that share the basic region/leucine zipper DNA binding motif and bind to the ATF/CRE consensus sequence TGACGTCA (see reviews by Hai et al. [19, 20]). Overwhelming evidence indicates that the expression of Atf3 is upregulated by a variety of signals, including some seemingly unrelated signals such as cytokines, nutrient deprivation, serum stimulation and calcium signalling (see review by Hai [21]). Given the broad spectrum of the stimuli that can induce ATF3, it appears that Atf3 is an 'adaptive-response' gene used by the cells to adapt to extraand/or intracellular changes. Borrowing a concept from the network theory, we suggest that ATF3 can be viewed as a hub in the biological networks to respond to signals perturbing homeostasis. Functionally, ATF3 is proapoptotic in pancreatic beta cells. Previously, we demonstrated that knockout $(\mathrm{KO})$ or knockdown of Atf3 protects islets or beta cells from stress-induced death [22, 23].

The deleterious effect of ATF3 in beta cells, combined with its general inducibility, prompted us to hypothesise that ATF3 is induced by signals encountered by the islets during transplantation, and that deletion of ATF3 would protect islets in a syngeneic transplantation model. In this model, donor mice have the same genetic background as recipient mice; thus the islets would not invoke the adaptive immune response, allowing us to investigate the roles of ATF3 in primary non-function. In this report, we present evidence supporting our hypothesis. We also present evidence that ATF3 upregulates the production of several pro-inflammatory cytokines and chemokine ( $\mathrm{C}-\mathrm{C}$ motif) ligand 2 (CCL2), a potent macrophage recruitment factor [24]. These results suggest that ATF3 contributes not only to apoptosis but also to an inflamed state of the islet grafts. Considering the deleterious effects of apoptosis and inflammation, our findings have significant implications for islet transplantation. 


\section{Methods}

Cell culture and treatments INS-1 and INS-r3 cells were grown as previously described [25]. Treatments were the following: IL-1 $\beta$ (5 ng/ml), TNF- $\alpha$ (30 ng/ml), IFN- $\gamma$ (125 ng/ml) and hypoxia $\left(1 \% \mathrm{O}_{2}, 5 \% \mathrm{CO}_{2}, 94 \% \mathrm{~N}_{2}\right)$.

Animals and adenoviruses Wild-type (WT) and Atf3 KO mice were detailed previously [22]. Animal experiments followed National Institutes of Health (NIH) guidelines (NIH publication no. 85-23) and were approved by the Ohio State University Laboratory Animal Resources. Adenoviruses were described previously [23].

Islet isolation and transplantation Islets isolated from agematched male mice (12-20 weeks, C57BL/6 background) as previously described [23] were incubated with RPMI containing $10 \%$ (vol./vol.) FBS (250 islets in $3 \mathrm{ml}$ ). Transplantations beneath the kidney capsule were carried out as previously described [26, 27] using 250-400 (as specified) freshly isolated islets.

Graft recovery Two days after transplantation, the graft was excised, placed into Trizol, vortexed (2 min) and rocked (overnight, $4^{\circ} \mathrm{C}$ ) prior to RNA extraction. For controls, similar-sized kidney capsules were excised from mice transplanted with PBS.

qRT-PCR, chromatin immunoprecipitation (ChIP) assay, immunoblotting and immunohistochemistry All procedures were as described before [23, 28], using PCR primers and antibodies listed in the Electronic supplementary material [ESM].

Cell viability analysis Islets were stained with propidium iodide (PI) (1 $\mu \mathrm{g} / \mathrm{ml})$ and Hoechst $33258(2 \mu \mathrm{g} / \mathrm{ml})$ followed by epifluorescence analyses using the NIH ImageJ Software as detailed in ESM Fig. 1.

Cell fractionation INS cells were permeabilised with $0.025 \%$ digitonin buffer (20 mmol/1 HEPES, $\mathrm{pH} 7.5$, $100 \mathrm{mmol} / 1 \mathrm{KCl}, 2.5 \mathrm{mmol} / 1 \mathrm{MgCl}_{2}, 250 \mathrm{mmol} / 1$ sucrose) on ice for $20 \mathrm{~min}$, tested by Trypan Blue to verify permeability, and centrifuged $(12,000 \times \mathrm{g}, 20 \mathrm{~min})$ to obtain the cytosolic fraction (supernatant fraction).

Glucose tolerance tests and insulin ELISA Glucose tolerance testing was as described previously [23] and insulin was measured by ELISA (Linco, St Charles, MO, USA) using tail or facial vein bleed.

Boyden chamber macrophage migration assay Bone marrow cells were isolated from the femur and differentiated into macrophages as described before [29]. Islet-conditioned medium was made by incubating 200 islets in $0.5 \mathrm{ml}$ medium for $48 \mathrm{~h}$. The cell migration assay was carried out as described previously [30] using $8 \times 10^{5}$ macrophages.

Semi-quantification of immunohistochemistry signals For caspase 3 activation, the NIH ImageJ program was used as follows: (1) delineate the islets region of interest (ROI) manually using the polygon selection tool; (2) threshold the image and convert to black and white; (3) count the positive pixels; (4) calculate the ratio of the signal pixels to the total pixels in the ROI. For macrophage recruitment, the ROI is the area within the kidney subcapsule that is not occupied by islets (see ESM Fig. 4).

Statistics All quantitative data are expressed as means \pm SEM, and comparisons made by Student's $t$ test unless otherwise indicated.

\section{Results}

The effects of Atf3 KO in islet isolation stress We examined whether Atf3 is expressed in the islets after isolation. Figure 1a shows that the level of Atf3 mRNA was the highest immediately after isolation and declined at 12-24 h. Figure $1 \mathrm{~b}$ is a representative immunoblot, confirming the induction of ATF3 in the WT but not KO islets. To test the potential significance of Atf3 expression, we compared WT and $\mathrm{KO}$ islets for their viability using PI coupled with Hoechst stain. Viable cells are not permeable to PI and will only be stained by Hoechst; however, dead cells will be stained by both PI and Hoechst. Figure 1c shows a representative picture with more PI stain in the WT than in the KO islets, indicating that ATF3 enhances cell death. Data quantification using the NIH ImageJ program (detailed in ESM Fig. 1) showed a higher percentage of PI-positive pixels in the WT than in the $\mathrm{KO}$ islets (Fig. 1d). Interestingly, both the WT and KO islets showed a decline in cell death over time, but the WT islets showed a slight rebound at the $48 \mathrm{~h}$ time point, paralleling an increase in ATF3 production at this time point.

Since ATF3 is a transcription factor, it most likely exerts its action, at least in part, by regulating downstream genes. We thus examined the expression of various candidate genes by qRT-PCR. We focused on two categories of genes: pro-apoptotic genes and genes involved in immunomodulation. The reason for testing immunomodulating genes is that, upon transplantation, the grafts induce immune responses from the hosts. Their ability to modulate the host responses greatly impacts the survival of the grafts. Although limited in scope, our screen showed higher expression of the following genes in the WT than in the 


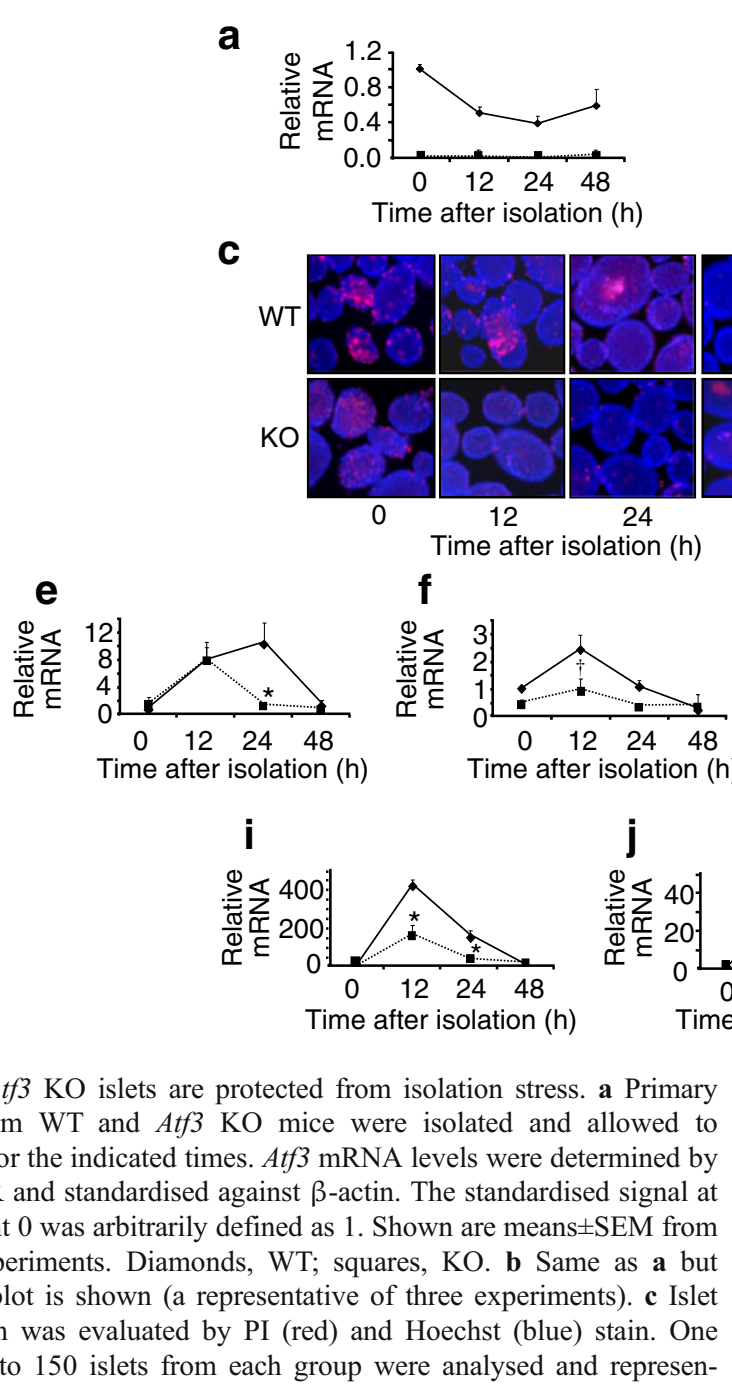

Fig. 1 Atf $3 \mathrm{KO}$ islets are protected from isolation stress. a Primary islets from WT and Atf3 $\mathrm{KO}$ mice were isolated and allowed to recover for the indicated times. Atf3 mRNA levels were determined by qRT-PCR and standardised against $\beta$-actin. The standardised signal at time point 0 was arbitrarily defined as 1 . Shown are means \pm SEM from three experiments. Diamonds, WT; squares, KO. b Same as a but immunoblot is shown (a representative of three experiments). c Islet cell death was evaluated by PI (red) and Hoechst (blue) stain. One hundred to 150 islets from each group were analysed and represen-

KO islets (Fig. 1e-j): Noxa (also known as Pmaipl) and Bnip3 (pro-apoptotic genes), and $\operatorname{Tnf} \alpha$ (also known as Tnf), Il-1 $\beta$ (also known as Illb), Il-6 (also known as Ilo) and $\mathrm{Ccl} 2$ (immunomodulating genes). The differences were statistically significant $(p<0.05)$ except for Bnip3 and $\mathrm{Ccl} 2$, which showed a consistent trend in three independent experiments but the $p$ value was $>0.05(0.13$ for Bnip3 and 0.08 for $C c l 2$ ). We also examined $C s f 1$, a macrophage recruitment factor, and $T f$, a factor whose expression contributes to islet loss in transplantation [31]. No difference in Csfl expression was observed between the WT and KO islets (Fig. 1k), and the difference for Tf was subtle (1.6 fold higher in WT, $p<0.03$ ). These results indicate that ATF3 contributes to the increased expression of certain pro-apoptotic and pro-inflammatory genes and would thus enhance islet apoptosis and inflammation. The notion of increased apoptosis is consistent with the higher cell death in the WT islets shown above (Fig. 1c). The b

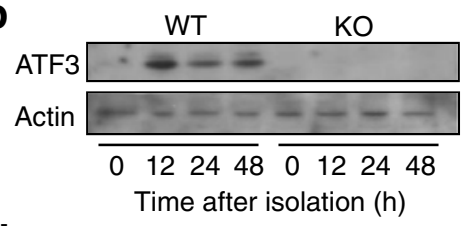

d

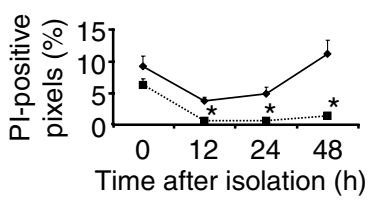

48
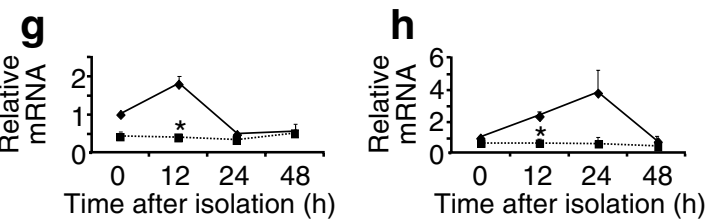

K

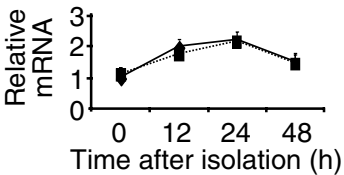

tative fields from each time point are shown. Scale bar, $400 \mu \mathrm{m}$. d Quantification of images in c. The $y$-axis is the percentage of Hoechstpositive pixels that are also PI-positive (see ESM Fig. 1 for detailed steps of image analysis). Diamonds, WT; squares, KO. ${ }^{*} p<0.001$ vs WT. e-k Same as in a except the indicated mRNAs for Noxa (e), Bnip3 (f), Il-1 $\beta$ (g), Tnfo (h), Il-6 (i), Ccl2 (j) and Csfl(k) were analysed. Shown are representative graphs from three experiments. Diamond, WT; squares, KO. ${ }^{*} p<0.05,{ }^{\dagger} p=0.13,{ }^{\star} p=0.08$ vs WT

functional consequences of ATF3 in inflammation will be addressed below (Fig. 9).

The effects of Atf3 $\mathrm{KO}$ in islet pro-inflammatory stress Upon transplantation, grafts face a barrage of attacks from the hosts. We examined the induction of ATF3 by proinflammatory cytokines, as a combination of either two cytokines (IL-1 $\beta+$ IFN- $\gamma$ ) or three cytokines (IL-1 $\beta+$ IFN$\gamma+\mathrm{TNF}-\alpha$ ). Islets were used $72 \mathrm{~h}$ after isolation, so that ATF3 levels in the WT islets were relatively low before induction. Figure 2 shows that ATF3 was induced by cytokines in the islets at both the mRNA (Fig. 2a) and protein (Fig. 2b) levels. Previously, we reported that Atf3 $\mathrm{KO}$ islets are partially protected from two cytokine-induced apoptosis as evidenced by the decrease in the cell population with sub-2N DNA content [22]. Consistent with the previous results, Atf $3 \mathrm{KO}$ islets had reduced levels of activated caspase 3 compared with WT islets (Fig. 2b). We 
a

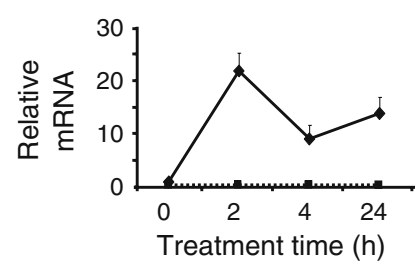

b

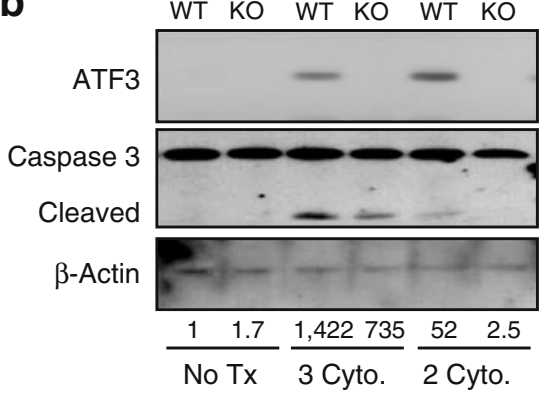

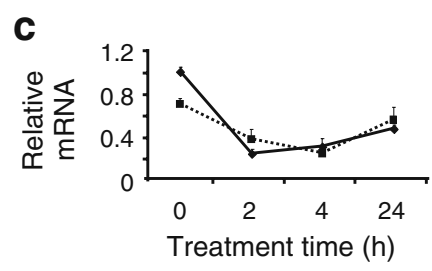

f

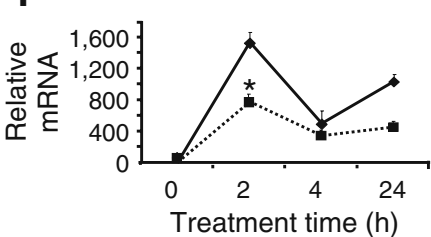

d

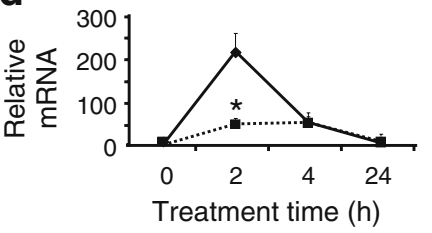

g

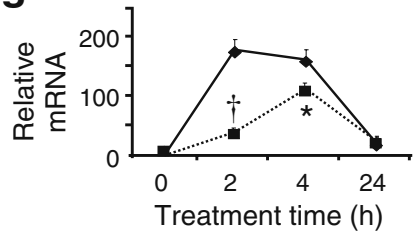

Fig. 2 Atf $3 \mathrm{KO}$ islets are protected from inflammatory cytokines. a Primary islets from WT and Atf $3 \mathrm{KO}$ mice were isolated and allowed to recover for $72 \mathrm{~h}$ prior to three-cytokine treatment (IL- $1 \beta$, TNF- $\alpha$ and IFN- $\gamma$ ). Atf3 mRNA levels were determined by qRT-PCR and analysed as in Fig. 1a. Shown are mean \pm SEM from three experiments. Diamonds, WT; squares, KO. b Islets prepared as in a were treated (Tx) with three cytokines (3 Cyto.) or two cytokines (2 Cyto., IL-1 $\beta$ and TNF- $\alpha$ ) and analysed by immunoblotting for the indicated proteins

then examined the expression of the candidate target genes described above. Figure $2 \mathrm{~d}-\mathrm{g}$ shows that the WT islets had higher expression of Tnf $\alpha, \mathrm{Il}-1 \beta, \mathrm{Il}-6$ and $\mathrm{Ccl} 2$ than the KO islets $(p<0.05)$, indicating that ATF3 also upregulates these genes (directly or indirectly) in the islets under the proinflammatory stress. No differences were observed for Bnip3 or Tf (Fig. 2c and data not shown). The data for Noxa were not reproducible in four repeated experiments (not shown). Since ATF3 is induced by the proinflammatory cytokines (TNF- $\alpha$, IL-1 $\beta$ ), its ability to induce the expression of Tnf $\alpha$ and $I l-1 \beta$ indicates a positive feedback loop.

The effects of Atf3 $\mathrm{KO}$ in islet hypoxia stress Upon removal from the donors, the islets are subjected to hypoxic stress until graft vascularisation in the host. We therefore examined the induction of ATF3 by hypoxia $\left(1 \% \mathrm{O}_{2}\right)$. Again, islets at $72 \mathrm{~h}$ after isolation were used to reduce the basal ATF3 level. Because the majority of islets started to disintegrate after 4-6 h under hypoxia, it was not possible to keep them continuously under this condition. We found that cyclic exposures of islets to hypoxia $(1 \%)$ and at $24 \mathrm{~h}$ after treatment. Shown is a representative of three experiments. The densitometry signals for cleaved caspase 3 were standardised against that for $\beta$-actin; the standardised signal for WT islets with no treatment was arbitrarily defined as 1 and the relative signals are indicated at the bottom of the blot. c-g Same as in a except the indicated mRNAs for Bnip3 (c), Tnf $\alpha$ (d), Il-1 $\beta$ (e), Il-6 (f) and Ccl2 (g) were analysed. Shown are representative graphs from four experiments. Diamonds, WT; squares, KO. ${ }^{*} p<0.05,{ }^{\dagger} p<0.02$ vs WT

normoxia $(20 \%)$ for $1 \mathrm{~h}$ each allows them to maintain overall integrity for $8 \mathrm{~h}$. At the end of four cycles, the islets start to lose their smooth boarders and appear unhealthy. We therefore terminated the experiments after four cycles of hypoxia and normoxia (Fig. 3a). Immunoblots indicated that ATF3 was induced at the end of all cycles, and Fig. 3b shows the data for cycles two and four. We also examined the induction of hypoxia-inducible factor 1 alpha subunit (HIF1- $\alpha$ ) as a positive control for the hypoxic conditions (Fig. 3b). PI coupled with Hoechst stain indicated reducing viability (more PI stain) over cycles of hypoxia and normoxia (Fig. 3c). As shown in the representative images, PI signals were always at the centre of the islets, consistent with the notion that the centre of the islets have least oxygen and are most vulnerable to hypoxic stress. This is in contrast to isolation stress, where PI signals started as scattered on the islet surface then focused in the centre of the islets (Fig. 1c), presumably because of the development of a hypoxic centre over time. We also quantified the signals by counting PI-positive pixels. Under hypoxia, the size of the islet affects the size of the hypoxic centre; the bigger the islets, the bigger the hypoxic centre. Thus, 
a

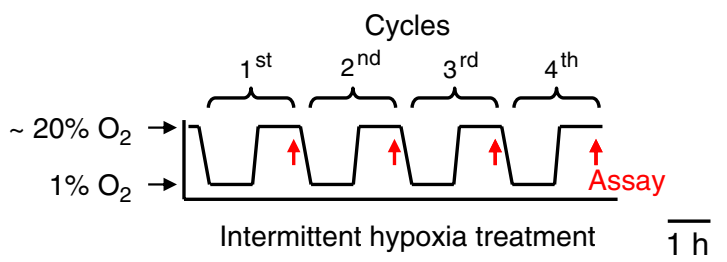

b

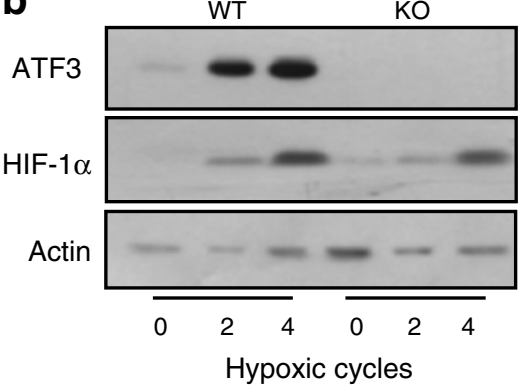

C

Control

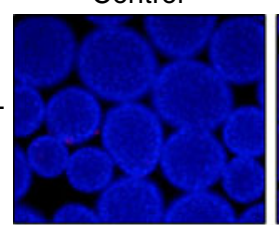

$2^{\text {nd }}$ Cycle

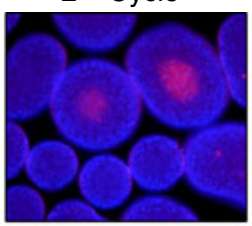

$4^{\text {th }}$ Cycle

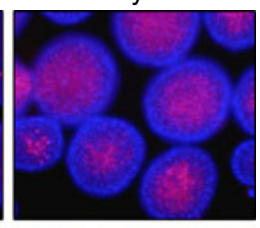

KO
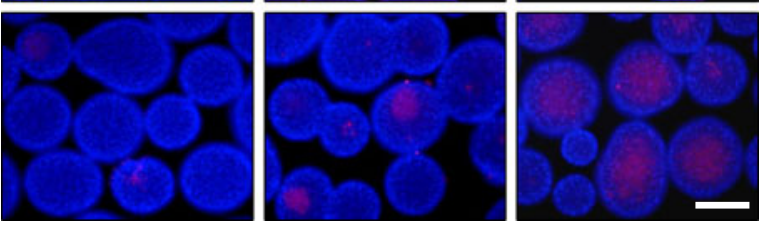

d

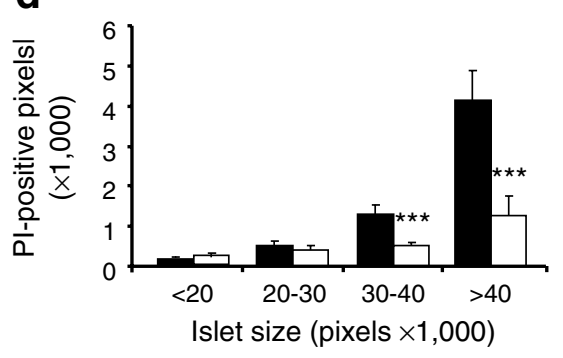

e

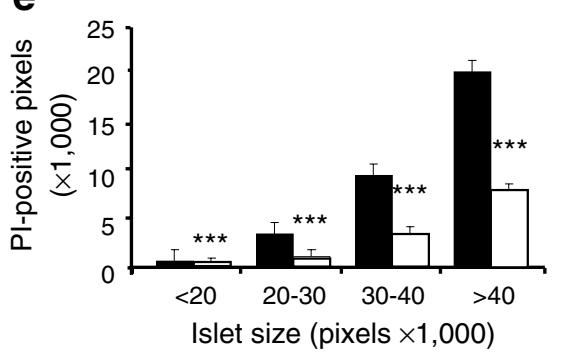

\section{f}

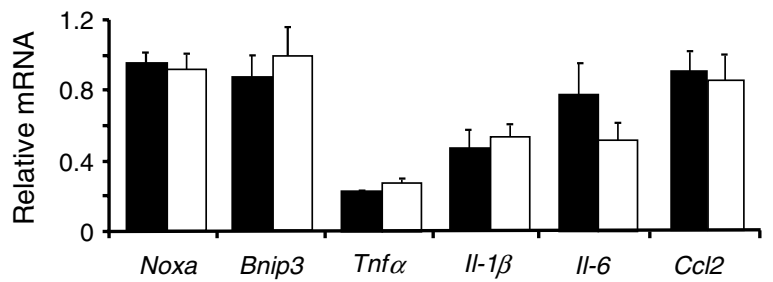

Fig. 3 Atf3 $\mathrm{KO}$ islets are protected from hypoxic stress. a A schematic of the intermittent hypoxia treatments: hypoxia $\left(1 \% \mathrm{O}_{2}\right)$ and normoxia $\left(20 \% \mathrm{O}_{2}\right)$ for $1 \mathrm{~h}$ each with assays at the end of each cycle (red arrows). b Primary islets from WT and Atf3 KO mice were isolated and allowed to recover for $72 \mathrm{~h}$ prior to hypoxia treatments. At the indicated cycles of treatments, the islets were analysed by immunoblot for the indicated proteins. Shown is a representative of three experiments. c Islet cell death was evaluated by PI (red) and Hoechst (blue) stain as in Fig. 1c, except that 250-300 islets were used in each group. Shown are representative fields from the indicated cycles. Scale bar, $200 \mu \mathrm{m}$. d, e Quantification of data in $\mathbf{c}$ for islets treated with two (d) or four (e) cycles of hypoxia. Islets were arbitrarily divided into four size groups and the percentage of PIpositive pixels (means \pm SEM) in each group are shown. WT, black bars; KO, white bars. ${ }^{* *} p<0.001$ vs WT. f Islets treated with two cycles of hypoxia were analysed for the indicated mRNAs as in Fig. 1e-k. The respective mRNA level under the unstressed condition was arbitrarily defined as 1 . Shown are average values from three experiments. WT, black bars; KO, white bars

cycles of hypoxia treatment (Fig. 3d), the differences between WT and KO islets were statistically significant in the larger islet groups, and after four cycles (Fig. 3e), the differences were significant for all four groups. Thus, WT islets were more vulnerable to hypoxic stress than $\mathrm{KO}$ islets. we plotted the number of PI-positive pixels against islet size (as measured by Hoechst stain). Figure $3 \mathrm{~d}$ and e show bar graphs of the PI-positive signals after arbitrarily dividing the islets into four groups: from small $(<20,000$ Hoechst-positive pixels) to big ( $>40,000$ pixels). After two 
We then examined the expression of various candidate genes in the islets after hypoxia stress. To our surprise, no genes showed statistically significant differences between WT and KO islets (Fig. 3f). Thus, under the hypoxia stress, ATF3 contributes to the reduced viability of the islets via a mechanism independent of the genes we have examined thus far.

The effects of ATF3 knockdown in beta cells Since the KO mice may develop compensation or adaptation that could complicate the phenotypes, we complemented the KO approach by knockdown. As shown in Fig. 4a, b, ATF3 was induced by cytokines in the INS-r3 beta cells and knocking it down reduced cytokine-induced caspase 3 activation. This result indicates that ATF3 promotes cytokine-induced beta cell apoptosis and is consistent with the above data using islets. As a step toward understanding the mechanisms of ATF3 action, we examined the effects of ATF3 knockdown on cytochrome $c$ release, a key step in apoptosis. Figure $4 \mathrm{c}$ shows that cytokine treatment induced cytochrome $c$ release and ATF3 knockdown reduced it.

Recruitment of ATF3 to the target promoters in the pancreatic beta cells Taken together, the above results indicate that ATF3 upregulates various target genes in a stress-dependent manner and contributes to the lower islet viability under all stresses examined. To address whether ATF3 directly regulates the target genes we reported here, we analysed their corresponding promoters by several programs and found multiple potential ATF3 binding sites on the promoters (ESM Fig. 2). Significantly, the majority of these sites are conserved in mouse and rat. To check the ability of ATF3 to bind to these sites in vivo, we carried out ChIP assays using INS-1 and INS-r3 cells infected with

Fig. 4 Atf3 knockdown protects beta cells from cytokine-induced caspase activation and cytochrome $c$ release. a INS-r3 cells were infected with adenovirus expressing control or Atf 3 shRNA (short hairpin RNA) for $24 \mathrm{~h}$ and treated (Tx) with cytokines (IL-1 $\beta$ and TNF$\alpha$ ) for the indicated time. Total cell lysates were analysed for the indicated proteins. A representative of three experiments is shown. b Blots from three experiments were quantified by densitometry for cleaved caspase 3 . The signals for cleaved caspase 3 were standardised against that for $\beta$-actin and the standardised signal for control knockdown at $0 \mathrm{~h}$ was arbitrarily defined as 1 . Means \pm SEM are shown. Black bars, control shRNA; white bars, Atf3 shRNA. $\dagger p<0.012$, $\ddagger p<$ 0.002. c INS-r3 cells with control or Atf3 knockdown were treated (Tx) with cytokines as in a for the indicated time. Cytosolic fraction (supernatant fraction) was separated from the nuclear/mitochondrial fraction (pellet), and analysed for the indicated proteins. Cyto. $c$, cytochrome $c$; Cox IV, subunit IV of cytochrome $c$ oxidase as a control for the pellet; Tubulin, a control for the supernatant fraction. The densitometry signals for Cyto. c were standardised against that for tubulin. The standardised signal for control knockdown at $0 \mathrm{~h}$ was arbitrarily defined as 1 and the relative signals are indicated at the bottom of the blot adenovirus expressing Atf3 or $\beta g a l$ (also known as Glb1) as a control. Comparable results were obtained from these two cell lines and the data from INS-1 cells are shown in Fig. 5. ATF3 indeed bound to the Noxa, Bnip3, Il-1 $\beta, \mathrm{Il}-6, \mathrm{Ccl} 2$ and Tnf $\alpha$ promoters. Several controls were carried out for the ChIP assay. The binding of ATF3 to the $\alpha$-actin (Acta2) or $\beta$-actin $(A c t b)$ promoter, which lacks recognisable ATF/ CRE sites, was examined. No binding was observed, suggesting (albeit not proving) that the signals we observed on the target genes were not caused by non-specific binding. In the same samples, PollI was found to bind to the promoter of $\beta$-actin (a beta cell gene) but not $\alpha$-actin (a non-beta cell gene), further validating the ChIP procedure (data not shown). For all experiments, the ChIP signals
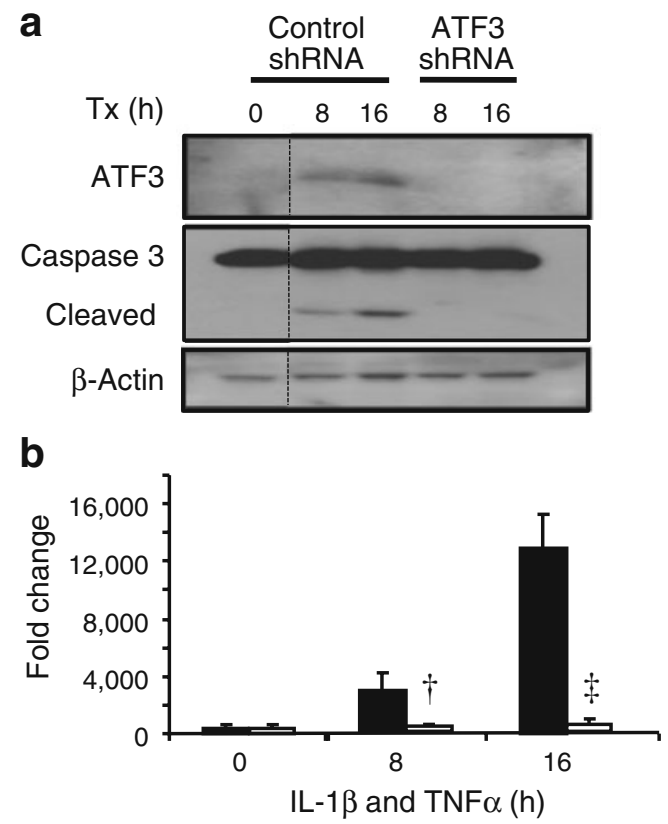

C
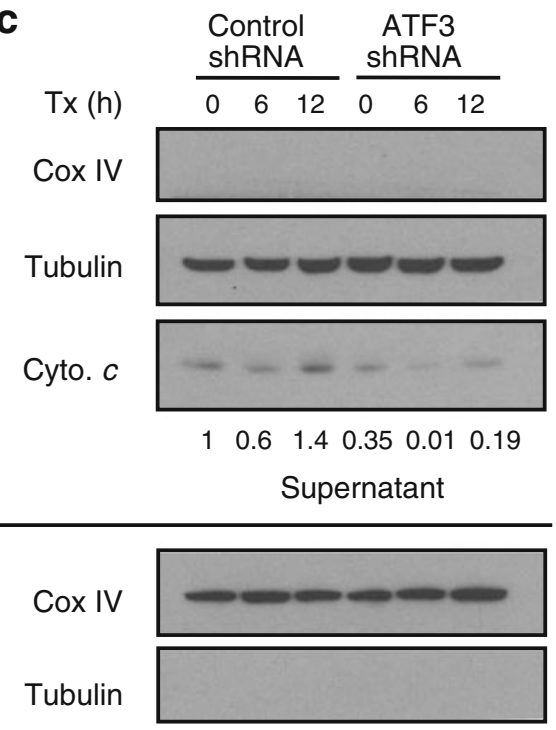

Pellet 


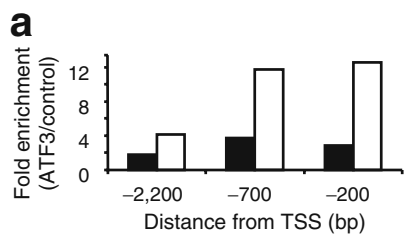

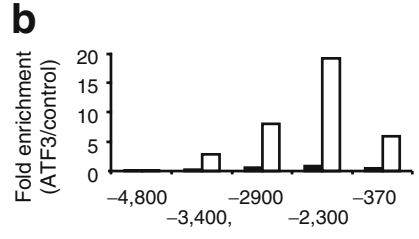

Distance from TSS (bp)

d

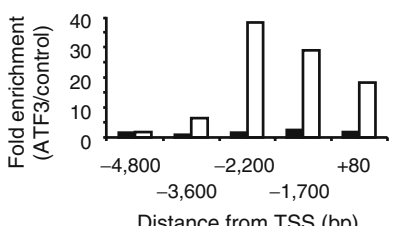

Distance from TSS (bp)
Distance from TSS (bp)

\section{e}

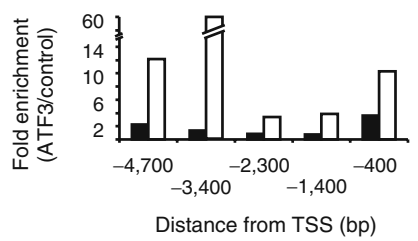

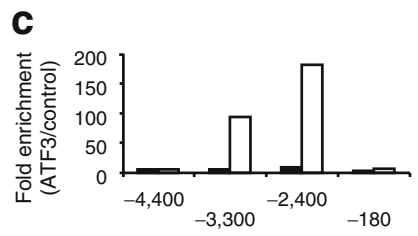

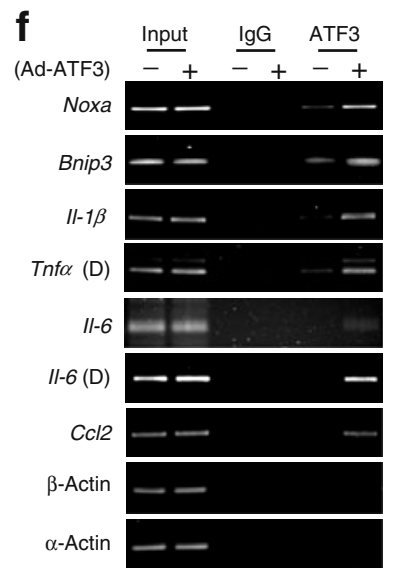

Fig. 5 Ectopically expressed ATF3 binds to the promoters of the proapoptotic and pro-inflammatory target genes. a-e INS-1 cells were infected with adenovirus expressing ATF3 (Ad-ATF3) or $\beta$ Gal (Ad$\beta \mathrm{Gal}$ ) for $24 \mathrm{~h}$ prior to ChIP analysis using primer pairs (schematics in ESM Fig. 2) for the potential binding sites at the indicated distance from the transcriptional start site (TSS) for Noxa (a), Bnip3 (b), Il-6 (c), $I l-1 \beta$ (d) and $C c l 2$ (e). For each primer pair, the ChIP signals were determined by qPCR and normalised to input chromatin. The normalised ChIP signals from the Ad-ATF3-infected cells were divided by that from the Ad- $\beta$ Gal-infected cells to obtain the 'fold enrichment'. Fold enrichment from immunoprecipitation using either IgG (black bars) or ATF3 antibodies (white bars) is shown. In four independent experiments, the pattern of binding (relative ATF3 recruitment to different sites on a given promoter) was largely the same, although the absolute fold enrichment varied to some extent from one experiment to another. A representative result is shown. $\mathbf{f}$ ChIP signals were analysed by agarose gel electrophoresis at the end of the linear phase of PCR reactions. The most proximal site for each promoter relative to the corresponding TSS was examined, except those indicated as distal (D). Tnf $\alpha$ (D): $-4,800$ bp; Il-6 (D): $-2,400 \mathrm{bp}$. Shown is a representative of four experiments were normalised against the input chromatin and the normalised signals from ATF3-producing cells were divided by that from $\beta$-galactosidase ( $\beta$ gal)-producing cells to obtain the fold enrichment. If the fold enrichment was the same for the ATF3 antibody and IgG (such as that at the $-4,800$ site on the $I l-1 \beta$ promoter), we interpreted it as background fold enrichment (thus no binding). Only if the fold enrichment using the ATF3 antibody was larger than that using IgG, did we interpret it as ATF3 binding. ESM Fig. 2 describes the arbitrary definition of low, medium and high binding. As summarised in ESM Fig. 2 and ESM Table 1, ATF3 bound to various sites on these promoters. We then asked whether the endogenous ATF3 binds to the promoters. We treated the INS-r3 cells with cytokines to increase the levels of ATF3 and examined its binding by ChIP. Figure 6a-f shows that ATF3 bound to the indicated sites of these promoters. We note that the binding of endogenous ATF3 was lower than that of the ectopically expressed ATF3 (using the above definition of binding), presumably owing to the lower levels of endogenous ATF3. We also examined the binding of the endogenous ATF3 to these promoters in islets. We treated the WT islets with cytokines immediately after isolation to increase ATF3 levels. Figure $6 \mathrm{~g}-1$ shows that ATF3 bound to these promoters, as evidenced by the higher ChIP signals using the ATF3 antibody than IgG. As a control, the Atf3 KO islets were used. No binding was observed except for Il-6, where some background binding was observed. Collectively, all the above ChIP results indicate that ATF3 binds (directly or indirectly) to the promoters examined.

The roles of ATF3 in a syngeneic islet transplantation model To test whether Atf $3 \mathrm{KO}$ islets would perform better than WT islets in transplantation, we implanted the islets under the kidney capsules of syngeneic WT mice. Mice were rendered diabetic by streptozotocin (STZ) 1 week prior to transplantation. At a saturating dose (400 islets), both WT and KO islets restored euglycaemia (ESM Fig. 3). At a marginal dose (250 islets), KO islets performed better than the WT islets as indicated by several criteria: (1) lower blood glucose levels from postoperative day (POD) 1 to POD28 (Fig. 7a); (2) higher per cent of euglycaemic mice from POD1 to POD28 (Fig. 7b); (3) better glucose tolerance test on POD28 (Fig. 7c); (4) smaller AUC in the glucose tolerance test (Fig. 7d); and (5) higher serum insulin level on POD28 (Fig. 7e). Removing the grafts at POD28 rendered the mice hyperglycaemic (Fig. 7a), confirming that the therapeutic effects were because of the transplanted islets. Since ATF3 is pro-apoptotic, we examined whether the $\mathrm{KO}$ islets had reduced apoptosis using activated caspase 3 as a marker by immunohistochemistry assay. The signals on POD5 were the highest among the days we examined (POD1, 3, 5 and 7). Semi- 
a

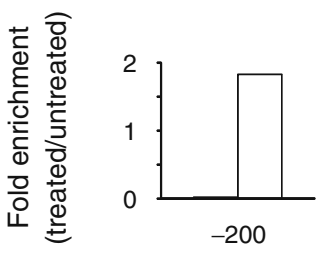

Distance from TSS (bp)

d

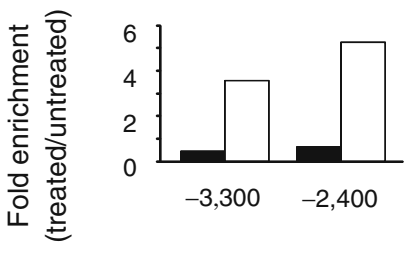

Distance from TSS (bp)

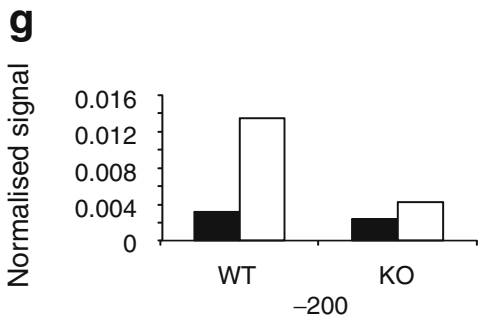

Distance from TSS (bp)

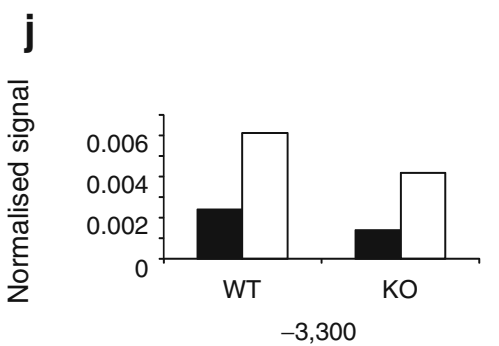

Distance from TSS (bp) b

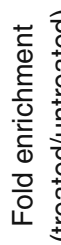

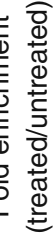

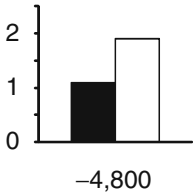

Distance from TSS (bp)

e

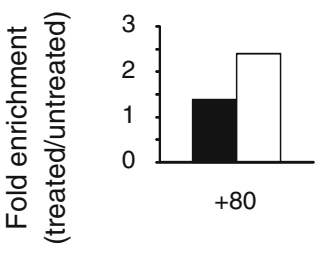

Distance from TSS (bp)

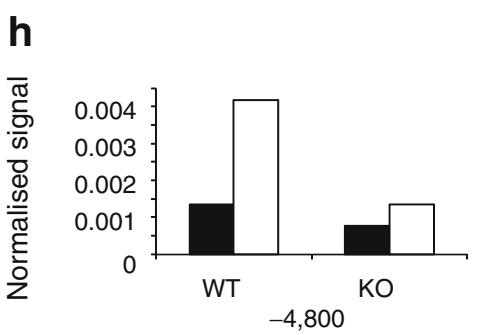

Distance from TSS (bp)

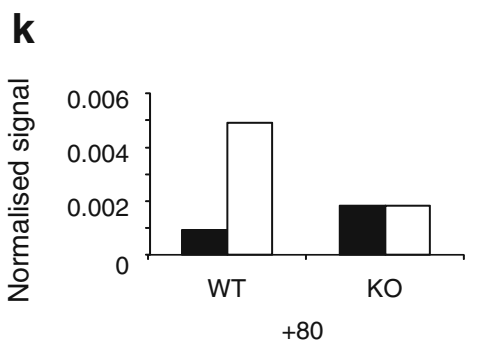

Distance from TSS (bp)
C

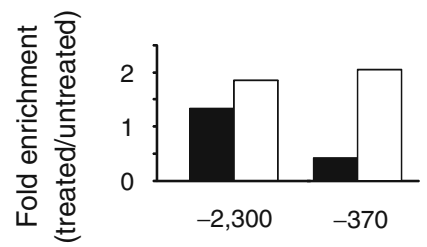

Distance from TSS (bp)

f

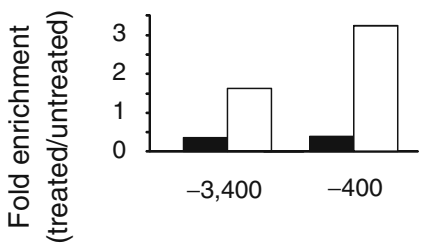

Distance from TSS (bp)

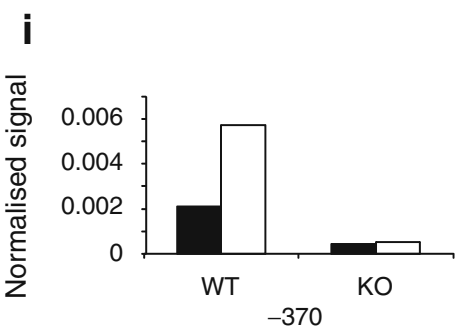

Distance from TSS (bp)

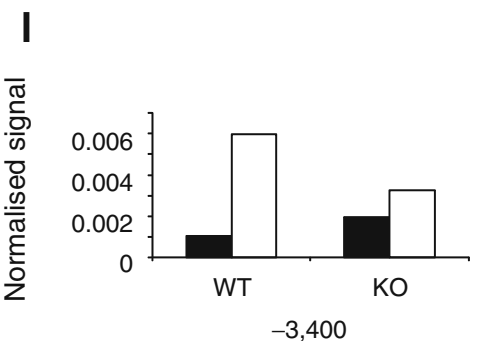

Distance from TSS (bp)
Fig. 6 Endogenous ATF3 binds to the promoters of the pro-apoptotic and pro-inflammatory target genes. a-f INS-r3 beta cells were untreated or treated with cytokines (IL- $1 \beta, \mathrm{TNF}-\alpha$ and IFN- $\gamma$ ), and ATF3 binding to the indicated promoters at $5 \mathrm{~h}$ after treatment was analysed by ChIP using the primers for the indicated sites for Noxa (a), Tnf $\alpha$ (b) Bnip3 (c), Il-6 (d), Il-1 $\beta$ (e) and Ccl2 (f). The normalised ChIP signals (against the input chromatin) from the treated cells were divided by that from the untreated cells to obtain the 'fold enrichment'. Fold enrichment from immunoprecipitation using either

quantification of the signals using the NIH ImageJ program (see "Methods") showed that KO islets had a lower percentage of area that stained positive for activated caspase 3 than the WT islets $(4.51 \pm 1.07 \%$ vs $15.69 \pm$ $3.12 \%$ in WT, multiple sections from four mice per group, $p<0.05$ ). Figure $7 \mathrm{f}$ shows some representative images. Parallel experiments using $\operatorname{IgG}$ as a negative control showed no signals. We also stained the graft sections for
IgG (black bars) or ATF3 antibodies (white bars) is shown. The experiments were carried out twice and the results from one experiment are shown. g-l WT or Atf3 KO islets immediately after isolation were treated with cytokines and ATF3 binding to the indicated promoters at $12 \mathrm{~h}$ after treatment was analysed by ChIP for Noxa (g), Tnf $\alpha$ (h) Bnip3 (i), Il-6 (j), Il-1 $\beta$ (k) and Ccl2 (l). The experiments were carried out twice and the results from one experiment are shown. Black bars, IgG; white bars, ATF3 antibody

ATF3 and confirmed its production in the WT but not KO islets. Insulin stains and haematoxylin plus eosin stains are shown for comparison.

To examine whether ATF3 contributes to the induction of the pro-apoptotic and pro-inflammatory genes described above, we recovered the grafts and analysed their mRNAs by qRT-PCR. Consistent with the in vitro data, WT islets had higher expression of Noxa, Tnf $\alpha, \mathrm{Il}-1 \beta, \mathrm{Il}-6$ and $\mathrm{Ccl} 2$ 

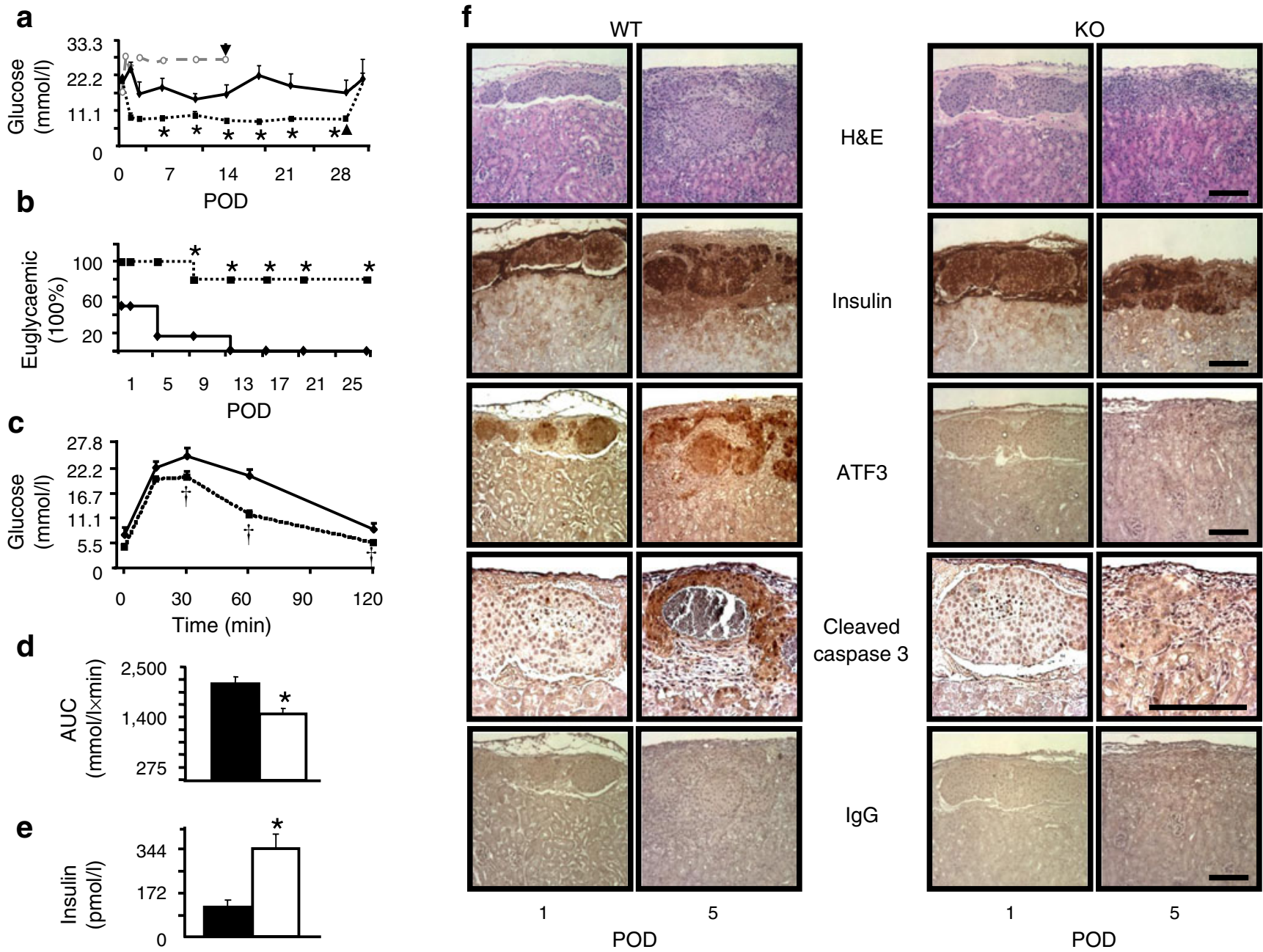

Fig. 7 Atf $3 \mathrm{KO}$ islets are protected from syngeneic transplantation stress. a C57BL/6 mice were rendered diabetic by STZ prior to receiving a marginal dose of $250 \mathrm{WT}$ or Atf $3 \mathrm{KO}$ islets. Non-fasted blood glucose levels were monitored from POD1 to POD28, when the grafts were removed (upward arrowhead). Diamond, WT; square, KO; circle, mock. $n=6$ for WT and $n=5$ for KO islet recipients $\left({ }^{*} p<0.05\right.$ vs WT). A mock transplant recipient (injected with PBS) died at POD14 as indicated (downward arrowhead). b Per cent of mice maintaining euglycaemia (blood glucose $<11 \mathrm{mmol} / \mathrm{l}$ ) through POD25 is shown. Diamond, WT; square, KO. ${ }^{*} p<0.05$ vs WT (Fisher's exact test). c Result from IPGTTs on POD28. Solid line, WT; dotted line, KO. $\uparrow p<0.05$ vs WT. d AUC from panel c. Black bar, WT; white bar, KO. ${ }^{*} p<0.005$ vs WT. e Fasting serum insulin on POD28. Black bar, $\mathrm{WT}$; white bar, KO. ${ }^{*} p<0.05$ vs WT. f Islets grafts at the indicated PODs were analysed by haematoxylin and eosin (H\&E) stain or immunohistochemistry using the indicated antibodies. Scale bar, $100 \mu \mathrm{m}$ than the $\mathrm{KO}$ islets (Fig. $8, p<0.05$ ), indicating that ATF3 contributes to their upregulation (directly or indirectly) in the grafts. Since CCL2 is a potent macrophage recruitment factor [24], we asked whether the WT and KO islets differ in their abilities to recruit macrophages by assaying the grafts for F4/80, a macrophage marker [32]. Figure 9a shows that macrophages were recruited to the grafts starting at POD2. Interestingly, they surrounded the grafts without infiltrating them. The recruitment continued to increase over time to POD7, when the experiments were terminated. To semi-quantify the signals, we used the NIH ImageJ program as detailed in the Methods. Figure $9 \mathrm{~b}$ shows that macrophage recruitment was lower in the $\mathrm{KO}$ than in the
WT grafts. Although the difference was only $10-20 \%$, they were statistically significant $(p<0.05)$, suggesting that ATF3 production in the WT islets contributes to their ability to recruit macrophages. To further test this idea, we compared the ability of the conditioned media derived from WT or KO islets (following the isolation stress) to enhance the motility of macrophages using the Boyden chamber migration assay. We isolated bone marrow cells from the femur and differentiated them into macrophages in vitro. We then placed the macrophages in the top half of the Boyden chamber and put the conditioned media from the islets in the bottom half, followed by measuring the macrophages on the underside of the membrane at $12 \mathrm{~h}$ 
a

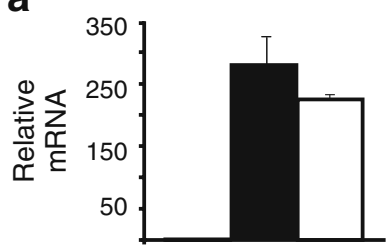

Ins

e

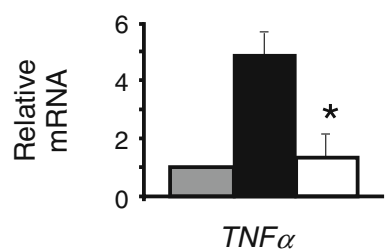

b

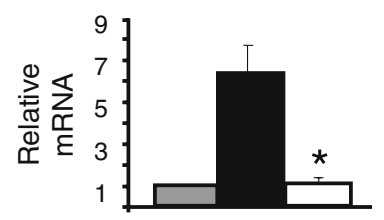

Atf3

f

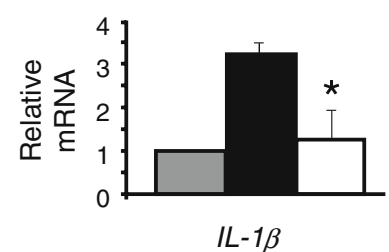

Fig. 8 The Atf $3 \mathrm{KO}$ islet grafts have reduced expression of pro-apoptotic and pro-inflammatory genes. WT and Atf $3 \mathrm{KO}$ grafts derived from 400 transplanted islets were recovered on POD2 and analysed for the indicated control (a, b), pro-apoptotic (c, d), or immunomodulatory (c-h)

C

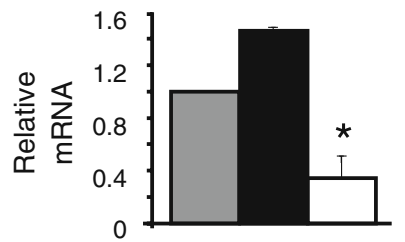

Noxa

g

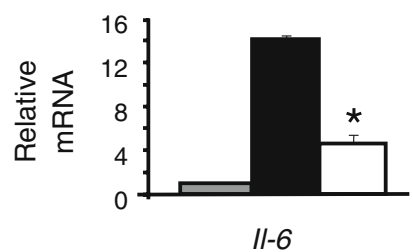

d

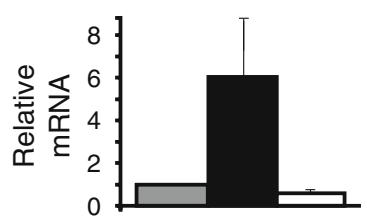

Bnip3

h

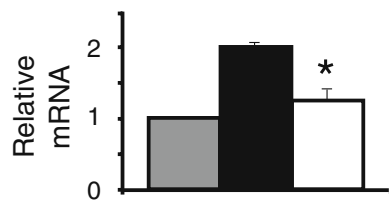

Ccl2

genes by qRT-PCR and standardised against $\beta$-actin. The standardised signal from mock transplant (injected with PBS) was arbitrarily defined as 1. Ins mRNA was used as a quality control for the grafts. Grey bars, mock transplant; black bars, WT; white bars, KO. ${ }^{*} p<0.05$ vs WT

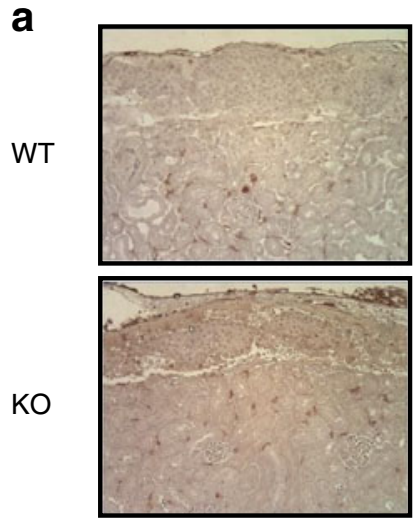

1

b

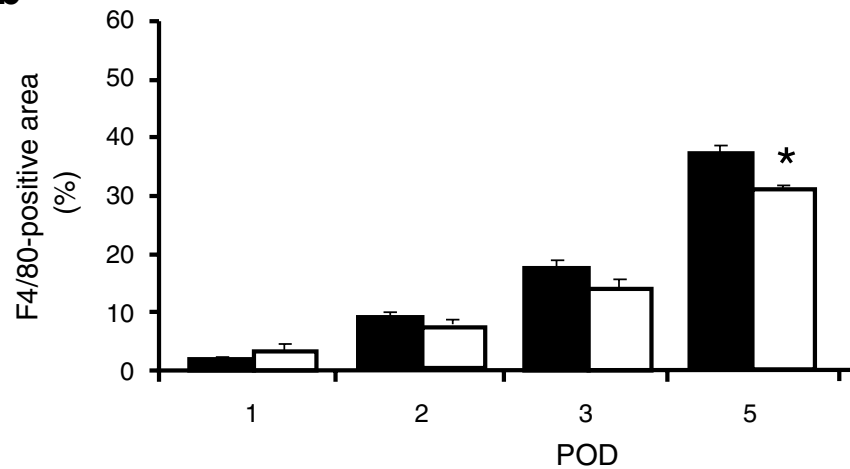

Fig. 9 Transplanted Atf3 $\mathrm{KO}$ islets promote less macrophage recruitment. a WT and $A t f 3 \mathrm{KO}$ grafts at the indicated PODs were analysed by immunohistochemistry for F4/80, a macrophage marker. Shown are representative fields from four mice each with 10-20 fields examined in each mouse. Scale bar, $100 \mu \mathrm{m}$. b Immunohistochemistry data in panel a were quantified using the methods detailed in the text and ESM Fig. 4. Black bars, WT; white bars, KO. ${ }^{*} p<0.05$ vs WT. c

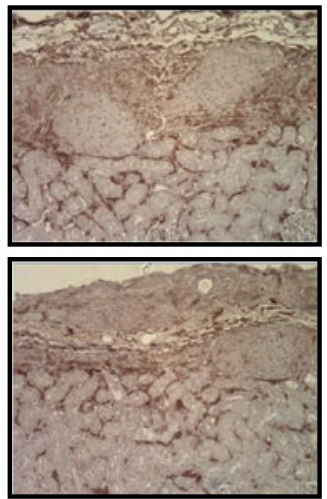

3

POD

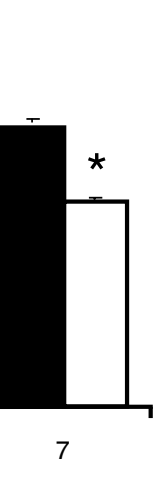

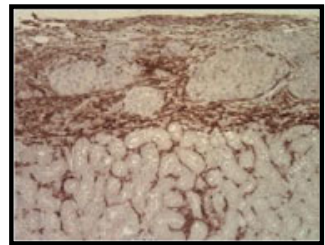
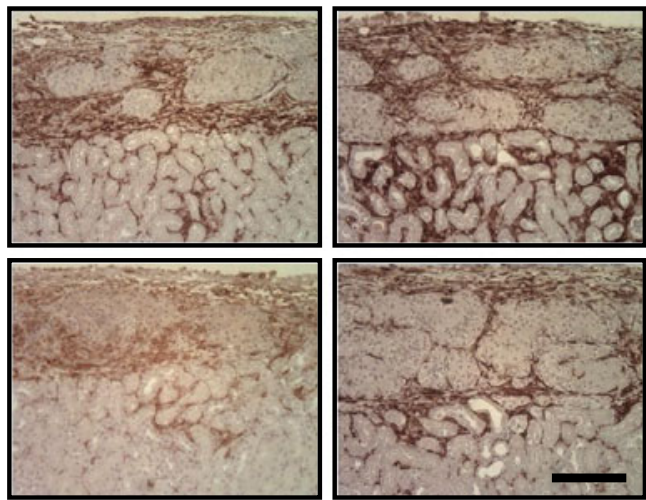

5

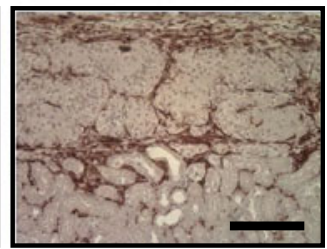

7

C

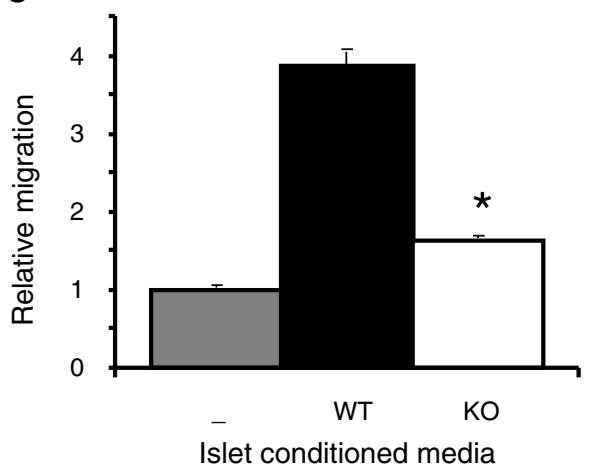

Bone marrow derived macrophages were analysed by the Boyden chamber migration assay using either media control (-) or conditioned media from the indicated islets in the bottom chambers. Migrated cells were stained by Crystal Violet and the signal from the media control (grey bar) was arbitrarily defined as 1 . The signals from the isletconditioned media were then standardised against the total RNA contents in the islets. ${ }^{*} p<0.05$ vs WT 
after incubation. Normal medium was used as a control and macrophage migration under this condition was arbitrarily defined as 1 . Figure $9 \mathrm{c}$ shows that conditioned media from the WT islets induced macrophages to migrate more efficiently than that from the $\mathrm{KO}$ islets. Thus, the in vitro migration assay supported the notion that ATF3 facilitates macrophage recruitment by the islets.

\section{Discussion}

In this report, we present evidence that ATF3 upregulates pro-apoptotic genes and genes involved in immunomodulation. Previously, we showed that ATF3 downregulates IRS2 [23], a potent pro-survival factor in beta cells, providing a mechanistic insight to the pro-apoptotic action of ATF3 in pancreatic beta cells [22, 23]. Results described in this report indicate that ATF3 upregulates the expression of Noxa, providing another potential explanation for the ability of ATF3 to promote beta cell death. Since apoptosis is a major reason for islet loss during transplantation, it is not surprising that the Atf $3 \mathrm{KO}$ islets performed better than WT islets in transplantation. Our results are consistent with previous reports that dampening apoptosis improves transplantation. However, our studies differ from previous reports in two key aspects. First, we dampened cell death by deleting a pro-apoptotic gene, rather than ectopically expressing anti-apoptotic genes, such as Tnfaip3 (also known as A20), Hgf, Bcl2, Akt, Irs2 and Xiap [26, 27, 33-37]. Since ATF3 is induced during the course of transplantation, our approach attenuates a naturally occurring deleterious event. Second, ATF3 deficiency also dampens inflammation, a benefit that has not been demonstrated by the other approaches. Since the inflammatory response has emerged as an important factor in islet graft rejection (below), this is a significant advantage.

The upregulation of $\mathrm{Ccl} 2$ by ATF3 has important implications. CCL2 is a chemokine and recruits macrophages [24]. Thus, its upregulation by ATF3 provides an explanation for the reduced macrophage recruitment in the KO grafts. Functionally, macrophage depletion in the recipients has been demonstrated to improve islet graft survival $[16,38]$. These results complement and further strengthen the idea that ATF3 deficiency is beneficial to the islet grafts. The upregulation of Tnf $\alpha, I l-1 \beta$ and $I l-6$ by ATF3 also has significant implications. These cytokines have been demonstrated to have deleterious effects on beta cells $[39,40]$ and contribute to primary non-function in islet transplantation [18, 41]. Since ATF3 itself is induced in the islets by TNF- $\alpha$ and IL- $1 \beta$, it forms a positive feedback loop to amplify these pro-inflammatory signals. The relevance of TNF- $\alpha$ in human islet transplantation is demonstrated by a clinical trial where the TNF- $\alpha$ receptor antagonist, in conjunction with a glucagon-like peptide-1 agonist, improved the Edmonton Protocol [8]. Thus, the ability of ATF3 to amplify TNF- $\alpha$ signal is of significance. We note that ATF3 has been shown to downregulate the expression of $T n f \alpha, I l-6$ and $I l-12 b$ (also known as $I l 12 b$ ) in macrophages upon lipopolysaccharide treatment [29, 42, 43]. Thus, ATF3 functions in macrophages to dampen, rather than enhance, the inflammatory responses (as shown here in islets). Explanations for this apparent discrepancy include the differences in cell types and stress signals.

Taken together, our results suggest a critical role for ATF3 in primary non-function. To the best of our knowledge, this is the first to demonstrate the protective effects of Atf3 KO in islet transplantation, and the first to implicate ATF3 in the ability of islet grafts to induce inflammatory reactions. In light of the emerging views that inflammation plays an important role in islet graft rejection [44, 45], our results suggest that ATF3 may be a therapeutic target to improve islet transplantation. The benefits of silencing Atf3 include not only the reduction of cellular decision to undergo cell death, but also the reduction of the ability of islets to provoke host inflammatory response.

Acknowledgements We thank L. Zhang for tips on kidney capsule injection and the Ohio State University Veterinary Medicine Histology Core for sectioning islet grafts. The work was supported in part by RO1 DK064938 (to T. Hai) and Ohio State Neuroscience Center Core grant (P30-NS045758) from the National Institute of Health.

Duality of interest The authors declare that there is no duality of interest associated with this manuscript.

\section{References}

1. Shapiro AM, Lakey JR, Ryan EA et al (2000) Islet transplantation in seven patients with type 1 diabetes mellitus using a glucocorticoidfree immunosuppressive regimen. N Engl J Med 343:230-238

2. Weir GC, Bonner-Weir S (1998) Islet transplantation as a treatment for diabetes. J Am Optom Assoc 69:727-732

3. Hogan A, Pileggi A, Ricordi C (2008) Transplantation: current developments and future directions; the future of clinical islet transplantation as a cure for diabetes. Front Biosci 13:1192-1205

4. Merani S, Shapiro AM (2006) Current status of pancreatic islet transplantation. Clin Sci (Lond) 110:611-625

5. Rother KI, Harlan DM (2004) Challenges facing islet transplantation for the treatment of type 1 diabetes mellitus. J Clin Invest 114:877-883

6. Witkowski P, Herold K (2007) Islet transplantation for type 1 diabetes - where should we go? Nat Clin Pract Endocrinol Metab $3: 2-3$

7. Naftanel MA, Harlan DM (2004) Pancreatic islet transplantation. PLoS Med 1:e58

8. Gangemi A, Salehi P, Hatipoglu B et al (2008) Islet transplantation for brittle type 1 diabetes: the UIC protocol. Am J Transplant $8: 1250-1261$

9. Jansson L, Carlsson PO (2002) Graft vascular function after transplantation of pancreatic islets. Diabetologia 45:749-763 
10. Davalli AM, Scaglia L, Zangen DH, Hollister J, Bonner-Weir S, Weir GC (1996) Vulnerability of islets in the immediate posttransplantation period. Dynamic changes in structure and function. Diabetes 45:1161-1167

11. Davalli AM, Scaglia L, Zangen DH, Hollister J, Bonner-Weir S, Weir GC (1995) Early changes in syngeneic islet grafts: effect of recipient's metabolic control on graft outcome. Transplant Proc 27:3238-3239

12. Davalli AM, Ogawa Y, Ricordi C, Scharp DW, Bonner-Weir S, Weir GC (1995) A selective decrease in the beta cell mass of human islets transplanted into diabetic nude mice. Transplantation 59:817-820

13. Davalli AM, Ogawa Y, Scaglia L et al (1995) Function, mass, and replication of porcine and rat islets transplanted into diabetic nude mice. Diabetes 44:104-111

14. Jonas JC, Sharma A, Hasenkamp W et al (1999) Chronic hyperglycemia triggers loss of pancreatic beta cell differentiation in an animal model of diabetes. J Biol Chem 274:14112-14121

15. Montana E, Bonner-Weir S, Weir GC (1997) Beta cell replication and mass in islet transplantation. Adv Exp Med Biol 426:421-427

16. Bottino R, Fernandez LA, Ricordi C et al (1998) Transplantation of allogeneic islets of Langerhans in the rat liver: effects of macrophage depletion on graft survival and microenvironment activation. Diabetes 47:316-323

17. Biarnes M, Montolio M, Nacher V, Raurell M, Soler J, Montanya E (2002) Beta-cell death and mass in syngeneically transplanted islets exposed to short- and long-term hyperglycemia. Diabetes 51:66-72

18. Wu Y, Han B, Luo H et al (2003) DcR3/TR6 effectively prevents islet primary nonfunction after transplantation. Diabetes 52:22792286

19. Hai T, Wolfgang CD, Marsee DK, Allen AE, Sivaprasad U (1999) ATF3 and stress responses. Gene Expr 7:321-335

20. Hai T, Hartman MG (2001) The molecular biology and nomenclature of the ATF/CREB family of transcription factors: ATF proteins and homeostasis. Gene 273:1-11

21. Hai $T$ (2006) The ATF transcription factors in cellular adaptive responses. In: Ma J (ed) Gene expression and regulation. Higher Education Press and Springer, Beijing, China and New York, USA, pp 322-333

22. Hartman MG, Lu D, Kim ML et al (2004) Role for activating transcription factor 3 in stress-induced beta-cell apoptosis. Mol Cell Biol 24:5721-5732

23. Li D, Yin X, Zmuda EJ et al (2007) The repression of IRS2 gene by ATF3, a stress-inducible gene, contributes to pancreatic B-cell apoptosis. Diabetes 57:635-644

24. Huang DR, Wang J, Kivisakk P, Rollins BJ, Ransohoff RM (2001) Absence of monocyte chemoattractant protein 1 in mice leads to decreased local macrophage recruitment and antigenspecific $\mathrm{T}$ helper cell type 1 immune response in experimental autoimmune encephalomyelitis. J Exp Med 193:713-726

25. Hohmeier HE, Mulder H, Chen G, Henkel-Rieger R, Prentki M, Newgard CB (2000) Isolation of INS-1-derived cell lines with robust ATP-sensitive $\mathrm{K}^{+}$channel-dependent and-independent glucose-stimulated insulin secretion. Diabetes 49:424-430

26. Grey ST, Longo C, Shukri T et al (2003) Genetic engineering of a suboptimal islet graft with A20 preserves beta cell mass and function. J Immunol 170:6250-6256

27. Garcia-Ocana A, Takane KK, Reddy VT, Lopez-Talavera J-C, Vasavada RC, Stewart AF (2003) Adenovirus-mediated hepatocyte growth factor expression in mouse islets improves pancreatic islet transplant performance and reduces beta cell death. J Biol Chem 278:343-351

28. Lu D, Wolfgang CD, Hai T (2006) Activating transcription factor 3, a stress-inducible gene, suppresses Ras-stimulated tumorigenesis. J Biol Chem 281:10473-10481

29. Khuu CH, Barrozo RM, Hai T, Weinstein SL (2007) Activating transcription factor 3 (ATF3) represses the expression of CCL4 in murine macrophages. Mol Immunol 44:1598-1605

30. Yin X, DeWille J, Hai T (2008) A potential dichotomous role of ATF3, an adaptive-response gene, in cancer development. Oncogene 27:2118-2127

31. Berman DM, Cabrera O, Kenyon NM et al (2007) Interference with tissue factor prolongs intrahepatic islet allograft survival in a nonhuman primate marginal mass model. Transplantation 84:308315

32. Leenen PJ, de Bruijn MF, Voerman JS, Campbell PA, van Ewijk W (1994) Markers of mouse macrophage development detected by monoclonal antibodies. J Immunol Methods 174:5-19

33. Fiaschi-Taesch NM, Berman DM, Sicari BM et al (2008) Hepatocyte growth factor enhances engraftment and function of nonhuman primate islets. Diabetes 57:2745-2754

34. Contreras JL, Bilbao G, Smyth C et al (2001) Gene transfer of the $\mathrm{Bcl}-2$ gene confers cytoprotection to isolated adult porcine pancreatic islets exposed to xenoreactive antibodies and complement. Surgery 130:166-174

35. Rao P, Roccisana J, Takane KK et al (2005) Gene transfer of constitutively active Akt markedly improves human islet transplant outcomes in diabetic severe combined immunodeficient mice. Diabetes 54:1664-1675

36. Hennige AM, Burks DJ, Ozcan U et al (2003) Upregulation of insulin receptor substrate- 2 in pancreatic beta cells prevents diabetes. J Clin Invest 112:1521-1532

37. Emamaullee JA, Rajotte RV, Liston P et al (2005) XIAP overexpression in human islets prevents early posttransplant apoptosis and reduces the islet mass needed to treat diabetes. Diabetes 54:2541-2548

38. Rossi L, Migliavacca B, Pierigé F et al (2008) Prolonged islet allograft survival in diabetic mice upon macrophage depletion by clodronate-loaded erythrocytes. Transplantation 85:648-650

39. Eizirik DL, Colli ML, Ortis F (2009) The role of inflammation in insulitis and beta-cell loss in type 1 diabetes. Nat Rev Endocrinol $5: 219-226$

40. Cnop M, Welsh N, Jonas JC, Jorns A, Lenzen S, Eizirik DL (2005) Mechanisms of pancreatic beta-cell death in type 1 and type 2 diabetes: many differences, few similarities. Diabetes 54 (Suppl 2):S97-S107

41. Gysemans C, Stoffels K, Giulietti A et al (2003) Prevention of primary non-function of islet xenografts in autoimmune diabetic NOD mice by anti-inflammatory agents. Diabetologia 46:1115-1123

42. Gilchrist M, Thorsson V, Li B et al (2006) Systems biology approaches identify ATF3 as a negative regulator of Toll-like receptor 4. Nature 441:173-178

43. Whitmore MM, Iparraguirre A, Kubelka L, Weninger W, Hai T, Williams BR (2007) Negative regulation of TLR-signaling pathways by activating transcription factor-3. J Immunol 179:3622-3630

44. Thomas FT, Hutchings A, Contreras J et al (2002) Islet transplantation in the twenty-first century. Immunol Res 26:289-296

45. Zhang YC, Pileggi A, Agarwal A et al (2003) Adeno-associated virusmediated IL-10 gene therapy inhibits diabetes recurrence in syngeneic islet cell transplantation of NOD mice. Diabetes 52:708-716 